Research Article

\title{
Conversion from Nonshockable to Shockable Rhythms and Out-of-Hospital Cardiac Arrest Outcomes by Initial Heart Rhythm and Rhythm Conversion Time
}

\author{
Wanwan Zhang, ${ }^{1}$ Shengyuan Luo, ${ }^{2,3,4}$ Daya Yang, ${ }^{2}$ Yongshu Zhang, ${ }^{1}$ Jinli Liao $\left(\mathbb{D},{ }^{1}\right.$ \\ Liwen Gu, ${ }^{1}$ Wankun Li, ${ }^{1}$ Zhihao Liu, ${ }^{1}$ Yan Xiong ${ }^{1},{ }^{1}$ and Ahamed Idris ${ }^{5}$ \\ ${ }^{1}$ Department of Emergency Medicine, The First Affiliated Hospital of Sun Yat-sen University, Guangzhou, Guangdong, China \\ ${ }^{2}$ Department of Cardiology, The First Affiliated Hospital of Sun Yat-sen University, Guangzhou, Guangdong, China \\ ${ }^{3}$ Department of Hypertension and Vascular Disease, The First Affiliated Hospital of Sun Yat-sen University, Guangzhou, \\ Guangdong, China \\ ${ }^{4}$ Department of Epidemiology, Johns Hopkins Bloomberg School of Public Health, Baltimore, MD, USA \\ ${ }^{5}$ Department of Emergency Medicine, University of Texas, Southwestern Medical Center, Dallas, TX, USA
}

Correspondence should be addressed to Yan Xiong; xiongyan@mail.sysu.edu.cn

Received 7 October 2019; Accepted 4 January 2020; Published 27 March 2020

Guest Editor: Shiyue Xu

Copyright (c) 2020 Wanwan Zhang et al. This is an open access article distributed under the Creative Commons Attribution License, which permits unrestricted use, distribution, and reproduction in any medium, provided the original work is properly cited.

\begin{abstract}
Background. The conversion from a nonshockable rhythm (asystole or pulseless electrical activity (PEA)) to a shockable rhythm (pulseless ventricular tachycardia or ventricular fibrillation) may be associated with better out-of-hospital cardiac arrest (OHCA) outcomes. There are insufficient data on the prognostic significance of such conversions by initial heart rhythm and different rhythm conversion time. Methods. Among 24,849 adult OHCA patients of presumed cardiac etiology with initial asystole or PEA in the Resuscitation Outcomes Consortium Cardiac Epidemiologic Registry (version 3, 2011-2015), we examined the association of shockable rhythm conversion with prehospital return of spontaneous circulation (ROSC), survival, and favorable functional outcome (modified Rankin Scale score $\leq 3$ ) at hospital discharge by initial rhythm and rhythm conversion time (time from cardiopulmonary resuscitation (CPR) initiation by emergency medical providers to first shock delivery), using logistic regression adjusting for key clinical characteristics. Results. Of 16,516 patients with initial asystole and 8,333 patients with initial PEA, 16\% and $20 \%$ underwent shockable rhythm conversions; the median rhythm conversion time was 12.0 (IQR: 6.7-18.7) and 13.2 (IQR: 7.0-20.5) min, respectively. No difference was found in odds of prehospital ROSC across rhythm conversion time, regardless of initial heart rhythm. Shockable rhythm conversion was associated with survival and favorable functional outcome at hospital discharge only when occurred during the first $15 \mathrm{~min}$ of CPR, for those with initial asystole, or the first $10 \mathrm{~min}$ of CPR, for those with initial PEA. The associations between shockable rhythm conversion and outcomes were stronger among those with initial asystole compared with those with initial PEA. Conclusions. The conversion from a nonshockable rhythm to a shockable rhythm was associated with better outcomes only when occurred early in initial nonshockable rhythm OHCA, and it has greater prognostic significance when the initial rhythm was asystole.
\end{abstract}

\section{Introduction}

The prognosis of out-of-hospital cardiac arrest (OHCA) remains poor [1-3]. OHCA patients with nonshockable rhythms (i.e., asystole and pulseless electrical activity (PEA)) are unlikely to benefit from an electrical defibrillation and suffer the worst outcomes [4]. Given that patients with nonshockable rhythms include the majority of presentations with the worst outcomes and represent the greatest opportunity to improve survival, the identification of prognostic factors in these patients is of clinical importance.

The conversion from a nonshockable rhythm to a shockable rhythm (i.e., pulseless ventricular tachycardia or ventricular fibrillation) has been shown to be associated with 
better short- or long-term outcomes in some, but not all OHCA populations [5-10]. In a previous meta-analysis involving 1,108,281 OHCA patients across 12 studies, we showed that the conversion from a nonshockable rhythm to a shockable rhythm and the subsequent electrical defibrillation attempt were associated with better outcomes only when the initial rhythm was asystole [5]. In a subgroup analysis, we found that the association between shockable rhythm conversion and 1-month favorable functional outcomes in patients with initial nonshockable rhythms tended to be weaker when rhythm conversion occurred late compared to early. In that analysis, however, only data from 2 studies, both conducted in Japan, were included $[11,12]$. Findings were not stratified by initial heart rhythm, and the full spectrum of OHCA outcomes was not examined. Further investigation is thus needed to thoroughly assess the prognostic significance of shockable rhythm conversion by initial rhythm and rhythm conversion time and across different outcomes in initial nonshockable rhythm OHCA.

Using data from the Resuscitation Outcomes Consortium (ROC) Cardiac Epidemiologic Registry (version 3, 2011- 2015), a North American population-based registry that included more than 60,000 EMS-treated OHCA events from 264 Emergency Medical Service (EMS) agencies and per-protocol ascertainments of multiple outcomes, we sought to thoroughly investigate the associations of conversion from a nonshockable rhythm to a shockable rhythm and prehospital return of spontaneous circulation (ROSC), survival, and favorable functional outcome at hospital discharge, stratified by initial heart rhythm and across the spectrum of rhythm conversion time in OHCA patients with initial nonshockable rhythms.

\section{Materials and Methods}

2.1. Study Design and Population. This study is a secondary analysis using data of the Resuscitation Outcomes Consortium (ROC) Cardiac Epidemiologic Registry from April 2011 to June 2015 (version 3). ROC is a network of clinical research of out-of-hospital cardiac arrest consisting of ten North Regional Centers (Ottawa, Toronto, Vancouver, Birmingham, Dallas, Pittsburgh, Milwaukee, Portland, Seattle/King County, and San Diego) across the United States and Canada and their respective EMS systems [13-16]. The present study population was derived from 67,204 patients who were treated by EMS providers in the ROC Cardiac Epidemiologic Registry from April 2011 to June 2015 (version 3). Patients with the following characteristics were included in the present study: age between 18 and 89 years, no existing do-not-resuscitate order, cardiac arrest of no obvious causes (presumed cardiac etiology), known initial rhythm, shock delivery status, and documented OHCA outcomes (Figure 1).

Study data were obtained from the National Institutes of Health (NIH) Biological Specimen and Data Repository Information Coordinating Center (https://biolincc.nhlbi. nih.gov/studies/roc_cardiac_epistry_3/?q=roc). The present study is a retrospective, observational analysis of this dataset approved by the Institutional Review Boards (IRBs) of ROC and NIH and then downloaded from the NIH website. The requirement of written informed consent was waived because of the nature of an anonymous dataset.

\section{Methods of Measurements}

The first recorded electrical defibrillation delivery during cardiopulmonary resuscitation (CPR) was used as the surrogate for the conversion from a nonshockable rhythm to a shockable rhythm. The time of shockable rhythm conversion was defined as the interval from the first chest compression by an EMS provider to the time of the first electrical shock delivery. Time-stamped data (hours: minutes: seconds) on chest compression initiation and shock deliveries were automatically recorded by monitor-defibrillators. All other covariables were ascertained based on standard ROC Cardiac Epidemiologic Registry protocols.

3.1. Outcomes. Three outcomes were assessed in this study: prehospital ROSC, survival to hospital discharge, and favorable functional outcome at hospital discharge, which was defined as a modified Rankin Scale score of $\leq 3$. Outcomes were ascertained by research personnel at each participating center through review of prehospital data streams, audio recordings, and hospital records. Modified Rankin Scale scores at hospital discharge were assigned using a standardized chart review instrument.

3.2. Data Analysis. Patient characteristics overall and stratified by initial rhythm (asystole or PEA) and categories of rhythm conversion time were summarized using descriptive statistics. The associations between shockable rhythm conversion (compared to no rhythm conversion) and outcomes were assessed using logistic regression with adjustment for age, sex, witness status (not witnessed vs. bystander witnessed vs. witnessed by EMS personnel), bystander CPR, location of OHCA (public vs. nonpublic), use of advanced airway, EMS response time, and use and dosage of epinephrine. Shockable rhythm conversion time was first modelled as a continuous variable, and cubic splines with knots at the $5^{\text {th }}, 35^{\text {th }}, 65^{\text {th }}$, and $95^{\text {th }}$ percentiles and the referent point at the $35^{\text {th }}$ percentile (conversion time$=10 \mathrm{~min}$ ), were used to visualize associations across conversion time. Rhythm conversion time was then categorized (<10 $\mathrm{min}, 10-15 \mathrm{~min}$, and $\geq 15 \mathrm{~min}$ ), and logistic regressions were repeated, comparing shockable rhythm conversion with nonshockable rhythm conversion, by conversion time categories. All analyses by continuous or categorical rhythm conversion time were stratified by initial rhythm (asystole or PEA). A two-sided $\alpha$-value of 0.05 was chosen as the cutoff for statistical significance. Statistical analyses were conducted using SPSS 20.0 (IBM Inc., Armonk, New York) and Stata 15.1 (StataCorp, College Station, Texas).

\section{Results}

4.1. Patient Characteristics. Of the 24,849 patients with initial nonshockable rhythm OHCA, 16,516 (66\%) had 


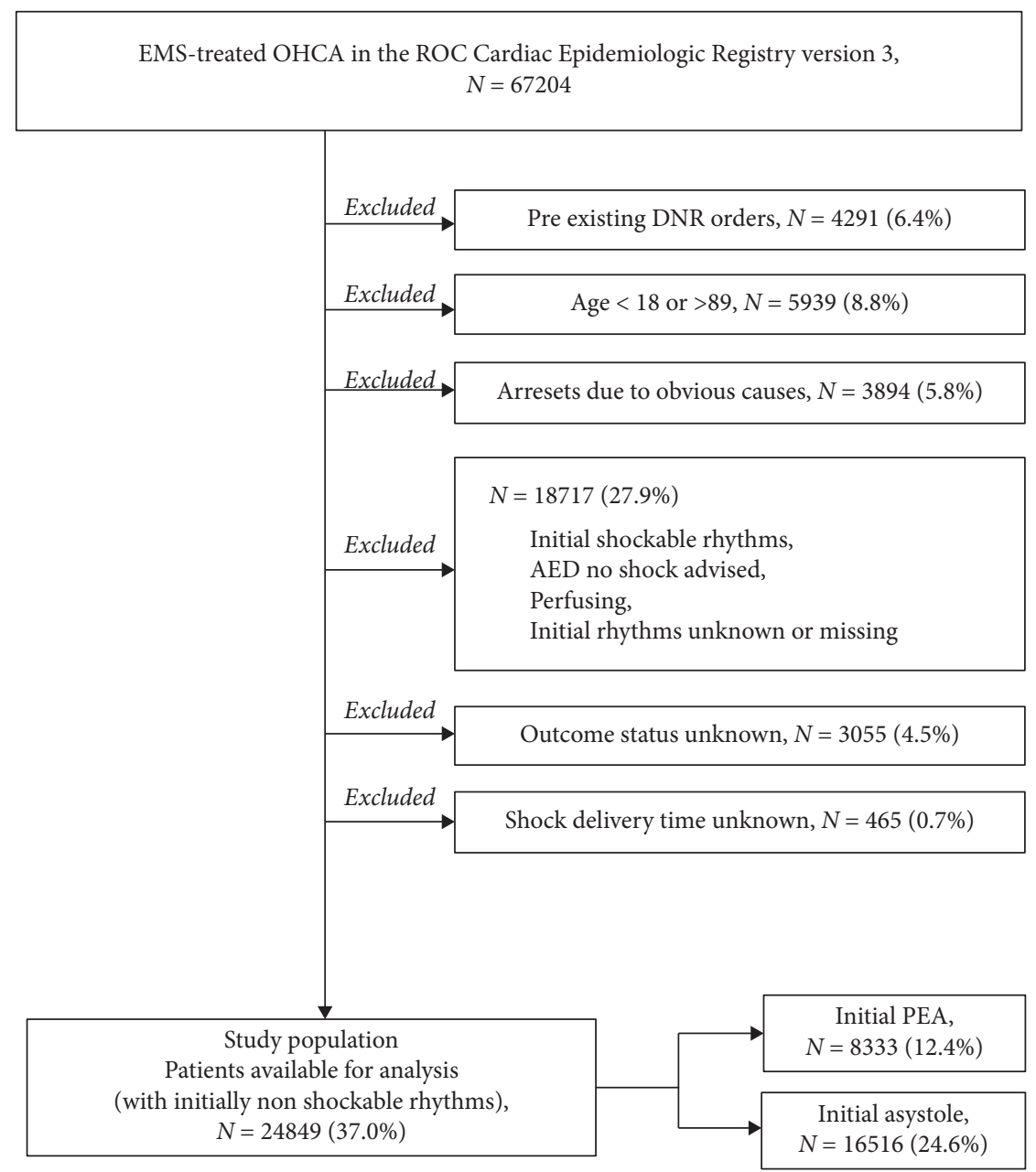

FIGURE 1: Study population selection process. EMS, Emergency Medical Services; OHCA, out-of-hospital cardiac arrest; ROC, Resuscitation Outcomes Consortium; DNR, do-not-resuscitate; AED, automatic external defibrillator; PEA, pulseless electrical activity; $N$, number.

initial asystole and 8,333 (34\%) had initial PEA (Table 1). Among patients with initial asystole and those with initial PEA, respectively, the median age was 66 (IQR: 54-77) and 70 (IQR: 59-79), 10,224 (62\%) and 5,120 (61\%) were men, $2,581(16 \%)$ and $1,655(20 \%)$ underwent shockable rhythm conversions, and the median rhythm conversion time was 12.0 (IQR: 6.7-18.7) and 13.2 (IQR: 7.0-20.5) min. Patient characteristics were comparable between those with initial asystole and those with initial PEA, except that the proportion of patients with OHCA witnessed by EMS personnel or bystander was higher among those with initial PEA. There was no statistical difference in patient characteristics across rhythm conversion time among those who underwent shockable rhythm conversions.

4.2. Shockable Rhythm Conversion and Prehospital ROSC. Among patients with initial asystole $(N=16,516)$ and those with initial PEA $(N=8,333)$, respectively, 3,361 $(20 \%)$ and 3,106 (37\%) had prehospital ROSC. Of the 1061, 567, and 953 patients with shockable rhythm conversions from initial asystole at $<10 \mathrm{~min}, 10-15 \mathrm{~min}$, and $\geq 15 \mathrm{~min}$ of CPR, 323 (30\%), 164 (29\%), and 322 (34\%), respectively, underwent prehospital ROSC. Of the 616, 330, and 705 patients with shockable rhythm conversions from initial PEA at $<10 \mathrm{~min}$, $10-15 \mathrm{~min}$, and $\geq 15 \mathrm{~min}$ of CPR, 238 (39\%), 123 (37\%), and 278 (39\%), respectively, underwent prehospital ROSC (Table 2). Using the $35^{\text {th }}$ percentile of rhythm conversion times $(10 \mathrm{~min})$ as the referent point, there was a trend towards increasing odds of prehospital ROSC with rhythm conversion time, when the initial rhythm was asystole and shockable rhythm conversion occurred within $10 \mathrm{~min}$ (Figure 2(a)), after adjustment for age, sex, witness status, bystander CPR, OHCA location, use of advanced airway, EMS response time, and use of epinephrine. After categorizing rhythm conversion time into $<10 \mathrm{~min}, 10-15 \mathrm{~min}$, and $\geq 15$ min of CPR and using nonshockable rhythm conversion as the reference, however, there was no observable difference in association between shockable rhythm conversion and prehospital ROSC by rhythm conversion time among those with asystole (Table 2). Among those with initial PEA, the association between shockable rhythm conversion and 


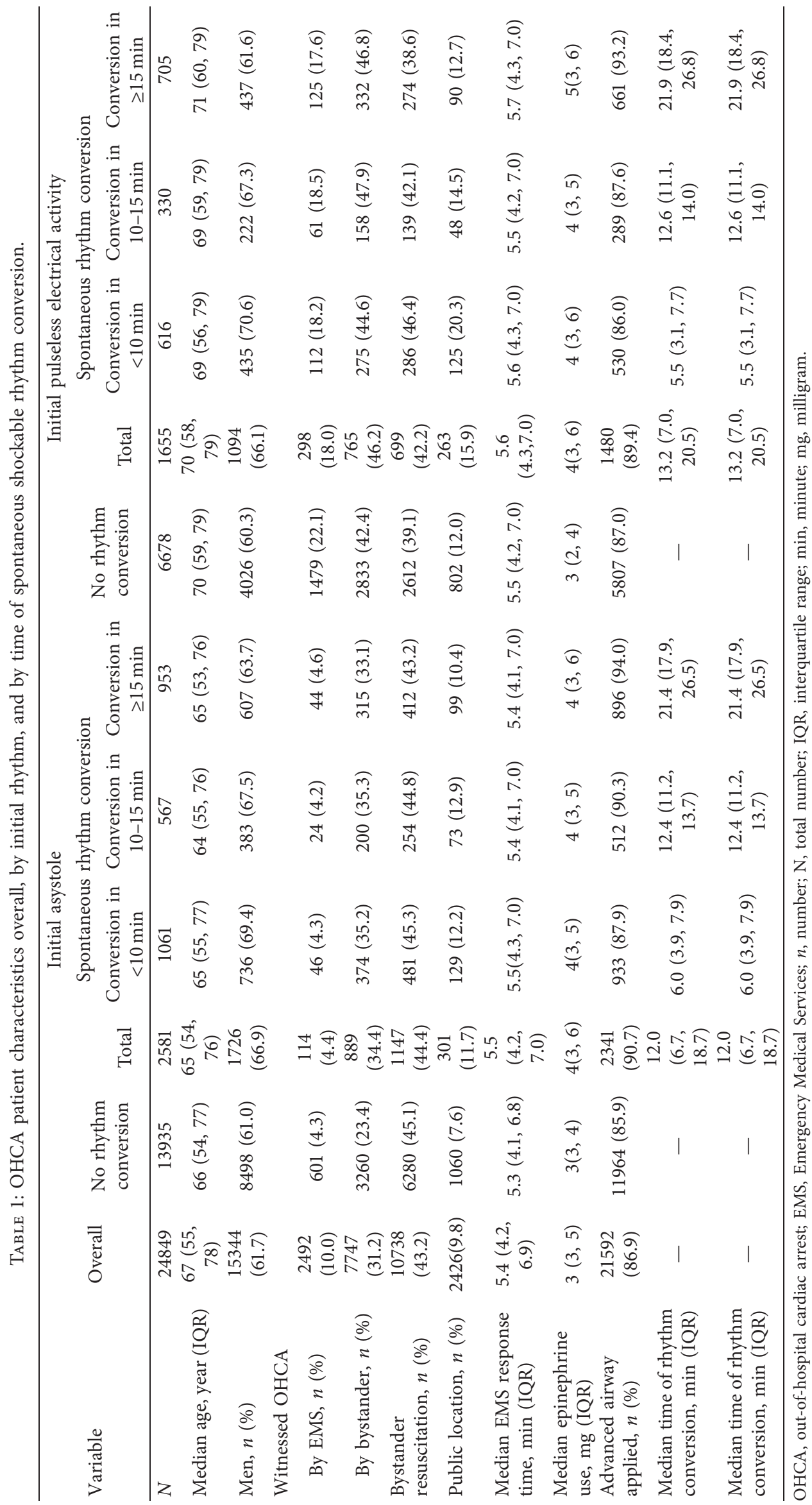


TABLE 2: Results from multivariable logistic regression analysis, assessing the associations of spontaneous rhythm conversion with prehospital ROSC, survival to hospital discharge, and favorable functional outcome in initial nonshockable rhythm OHCA stratifying by time of spontaneous shockable rhythm conversion.

\begin{tabular}{|c|c|c|c|c|c|c|c|}
\hline & \multirow{2}{*}{$\begin{array}{c}N \\
\text { total }\end{array}$} & \multicolumn{2}{|c|}{ Prehospital ROSC } & \multicolumn{2}{|c|}{ Survival to hospital discharge } & \multicolumn{2}{|c|}{$\begin{array}{c}\text { Favorable functional outcome at } \\
\text { hospital discharge }\end{array}$} \\
\hline & & $\begin{array}{c}N \text { of events } \\
\text { (proportion, \%) }\end{array}$ & $\begin{array}{l}\text { OR }(95 \% \\
\text { CI })\end{array}$ & $\begin{array}{c}N \text { of events } \\
\text { (proportion, \%) }\end{array}$ & $\begin{array}{l}\text { OR }(95 \% \\
\quad \text { CI })\end{array}$ & $\begin{array}{c}N \text { of events } \\
\text { (proportion, \%) }\end{array}$ & $\begin{array}{l}\text { OR }(95 \% \\
\text { CI })\end{array}$ \\
\hline \multicolumn{8}{|l|}{ Initial asystole } \\
\hline $\begin{array}{l}\text { No spontaneous } \\
\text { rhythm conversion }\end{array}$ & 13935 & $2552(18.2)$ & Reference & $118(0.8)$ & Reference & $47(0.3)$ & Reference \\
\hline $\begin{array}{l}\text { Spontaneous } \\
\text { conversion in } \\
<10 \text { min }\end{array}$ & 1061 & $323(30.4)$ & $\begin{array}{l}1.93(1.67 \\
2.23)\end{array}$ & $34(3.2)$ & $\begin{array}{l}4.39(2.95 \\
\quad 6.53)\end{array}$ & $14(1.3)$ & $\begin{array}{l}4.28(2.32 \\
\quad 7.89)\end{array}$ \\
\hline $\begin{array}{l}\text { Spontaneous } \\
\text { conversion in } \\
10-15 \text { min }\end{array}$ & 567 & $164(28.9)$ & $\begin{array}{l}1.76(1.45 \\
2.13)\end{array}$ & $12(2.1)$ & $\begin{array}{l}3.05(1.65 \\
5.62)\end{array}$ & $7(1.2)$ & $\begin{array}{l}4.38(1.94 \\
\quad 9.90)\end{array}$ \\
\hline $\begin{array}{l}\text { Spontaneous } \\
\text { conversion in } \\
\geq 15 \mathrm{~min}\end{array}$ & 953 & $322(33.8)$ & $\begin{array}{l}2.23(1.92 \\
2.59)\end{array}$ & $9(0.9)$ & $\begin{array}{l}1.60(0.80 \\
3.20)\end{array}$ & $2(0.2)$ & $\begin{array}{l}0.90(0.22 \\
\quad 3.74)\end{array}$ \\
\hline \multicolumn{8}{|c|}{ Initial pulseless electrical activity } \\
\hline $\begin{array}{l}\text { No spontaneous } \\
\text { rhythm conversion }\end{array}$ & 6678 & $2467(36.9)$ & Reference & $238(3.6)$ & Reference & $126(1.9)$ & Reference \\
\hline $\begin{array}{l}\text { Spontaneous } \\
\text { conversion in } \\
<10 \mathrm{~min}\end{array}$ & 616 & $238(38.6)$ & $\begin{array}{l}1.26(1.06 \\
1.50)\end{array}$ & $34(5.5)$ & $\begin{array}{l}2.09(1.42 \\
3.08)\end{array}$ & $20(3.2)$ & $\begin{array}{l}2.26(1.37 \\
3.75)\end{array}$ \\
\hline $\begin{array}{l}\text { Spontaneous } \\
\text { conversion in } \\
10-15 \text { min }\end{array}$ & 330 & $123(37.3)$ & $\begin{array}{l}1.15(0.91 \\
1.45)\end{array}$ & $12(3.6)$ & $\begin{array}{l}1.50(0.82 \\
2.77)\end{array}$ & $3(0.9)$ & $\begin{array}{l}0.72(0.23 \\
2.33)\end{array}$ \\
\hline $\begin{array}{l}\text { Spontaneous } \\
\text { conversion in } \\
\geq 15 \mathrm{~min}\end{array}$ & 705 & $278(39.2)$ & $\begin{array}{l}1.32(1.12 \\
1.56)\end{array}$ & $11(1.6)$ & $\begin{array}{l}0.88(0.47 \\
1.65)\end{array}$ & $4(0.6)$ & $\begin{array}{c}0.67(0.24 \\
1.85)\end{array}$ \\
\hline
\end{tabular}

Covariables in regression models include age, sex, witnessed OHCA (by EMS vs. bystander vs. not), bystander CPR, location of OHCA (public vs. not), use of advanced airway, Emergency Medical Services response time, and dose of epinephrine administered. Favorable functional outcome at hospital discharge is defined as a Modified Rankin Scale score of $\leq 3$. OHCA, out-of-hospital cardiac arrest; EMS, Emergency Medical Services; CPR, cardiopulmonary resuscitation; $\mathrm{N}$, number; min, minute; $\mathrm{OR}$, odds ratio.

prehospital ROSC was weaker compared to those with initial asystole and did not differ by rhythm conversion time.

4.3. Shockable Rhythm Conversion and Survival to Hospital Discharge. Among patients with initial asystole $(N=16,516)$ and those with initial PEA $(N=8,333)$, respectively, $173(1 \%)$ and 295 (4\%) survived to hospital discharge. Of the 1061, 567 , and 953 patients with shockable rhythm conversions from initial asystole at $<10 \mathrm{~min}, 10-15 \mathrm{~min}$, and $\geq 15 \mathrm{~min}$ of CPR, 34 (3\%), $12(2 \%)$, and $9(1 \%)$, respectively, survived to hospital discharge. Of the 616, 330, and 705 patients with shockable rhythm conversions from initial PEA at $<10 \mathrm{~min}$, $10-15 \mathrm{~min}$, and $\geq 15 \mathrm{~min}$ of CPR, 34 (6\%), 12 (4\%), and 11 (2\%), respectively, survived to hospital discharge. Using the $35^{\text {th }}$ percentile of rhythm conversion times $(10 \mathrm{~min})$ as the referent point, there was a linear trend towards decreasing odds of survival to hospital discharge with rhythm conversion time for both patients with initial asystole and those with initial PEA, adjusting for covariates (Figure 2(b)). After categorizing rhythm conversion time into $<10 \mathrm{~min}$, 10-15 min, and $\geq 15$ min of CPR, higher odds of survival to hospital discharge was observed with shockable rhythm conversion, when the initial rhythm was asystole and shockable rhythm conversion occurred within the first 10 min (odds ratio (OR) 4.39; 95\% confidence interval (CI): $2.95,6.53$ ) or $10-15$ min of CPR (OR 3.05; 95\% CI: 1.65 , 5.62), or when the initial rhythm was PEA and shockable rhythm conversion occurred within the first 10 min of CPR (OR 2.09; 95\% CI: 1.42, 3.08).

4.4. Shockable Rhythm Conversion and Favorable Functional Outcome. Among patients with initial asystole $(N=16,516)$ and those with initial PEA $(N=8,333)$, respectively, 70 $(0.4 \%)$ and 153 (2\%) had a favorable functional outcome at hospital discharge. Of the 1061, 567, and 953 patients with shockable conversions from initial asystole at $<10 \mathrm{~min}$, $10-15 \mathrm{~min}$, and $\geq 15 \mathrm{~min}$ of CPR, 14 (1\%), 7 (1\%), and 2 $(0.2 \%)$, respectively, had a favorable functional outcome at discharge. Of the 616, 330, and 705 patients with shockable conversions from initial PEA at $<10 \mathrm{~min}, 10-15 \mathrm{~min}$, and $\geq 15$ min of CPR, 20 (3\%), 3 (1\%), and 4 (1\%), respectively, had a favorable functional outcome at discharge. Using the $35^{\text {th }}$ percentile of rhythm conversion times $(10 \mathrm{~min})$ as the referent point, there was a trend towards decreasing odds of favorable functional outcome at discharge with shockable rhythm conversion time, most prominently when occurred 


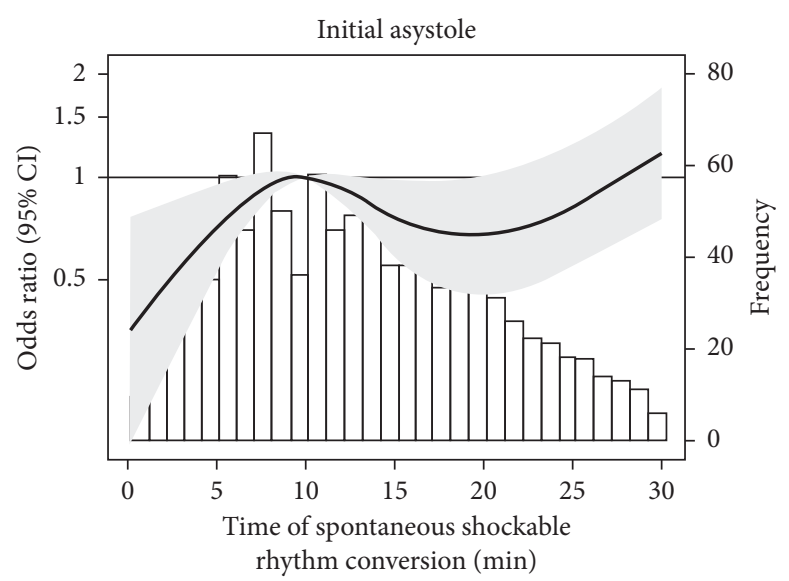

(a)

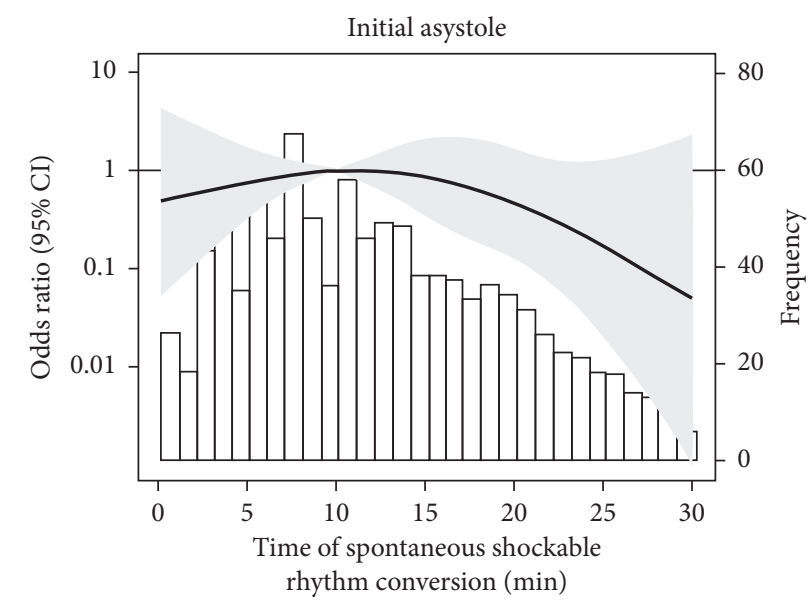

(c)

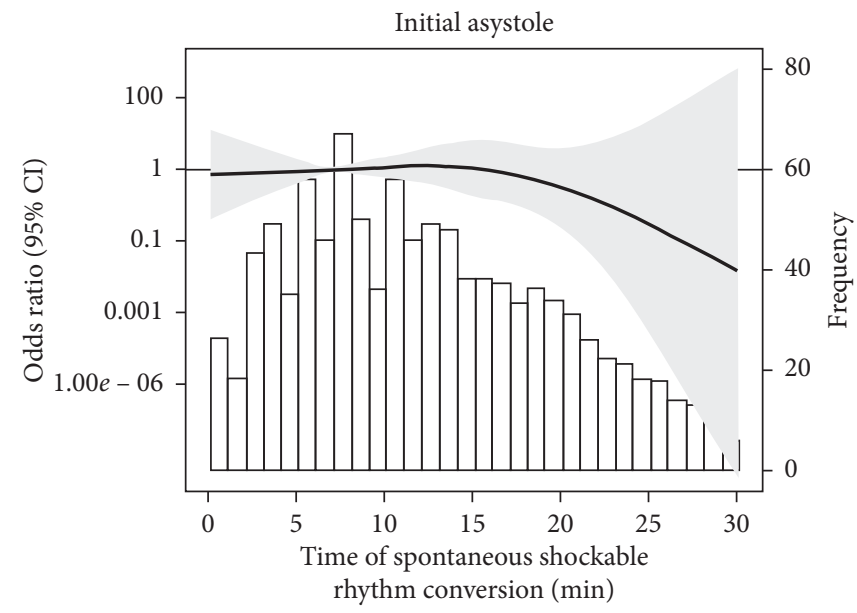

(e)

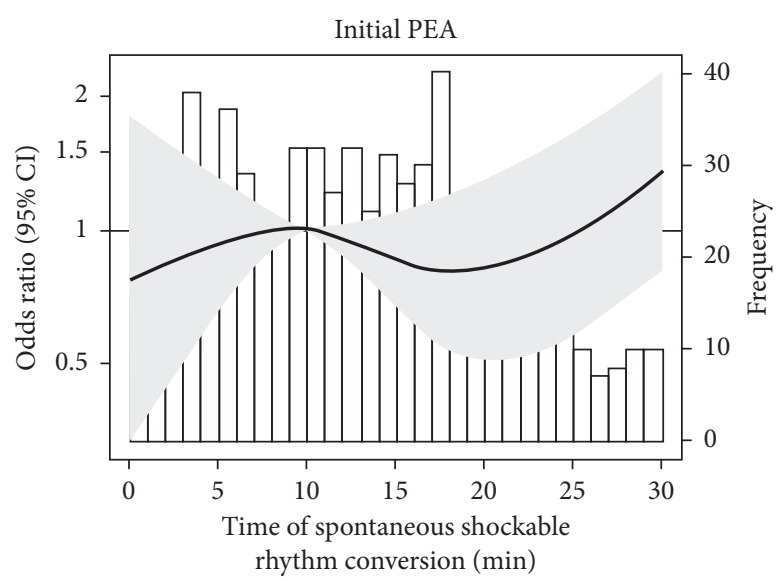

(b)

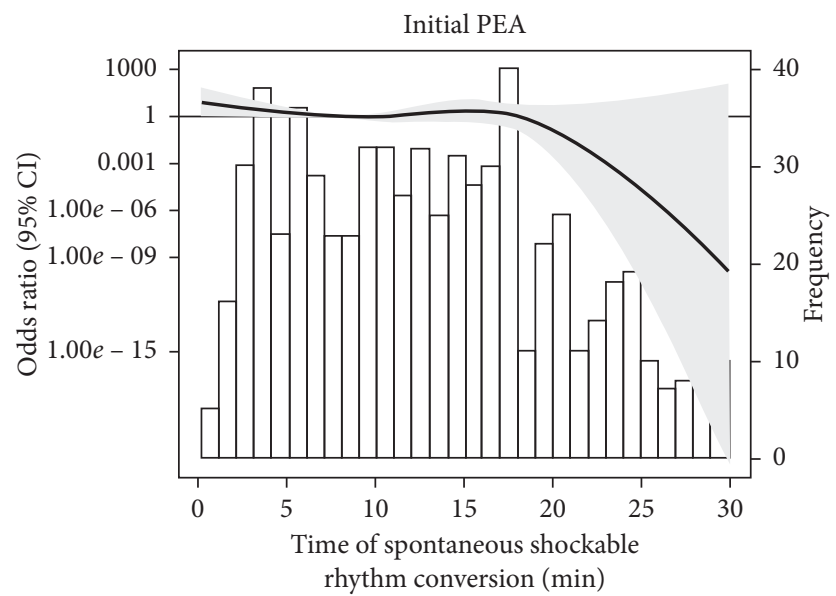

(d)

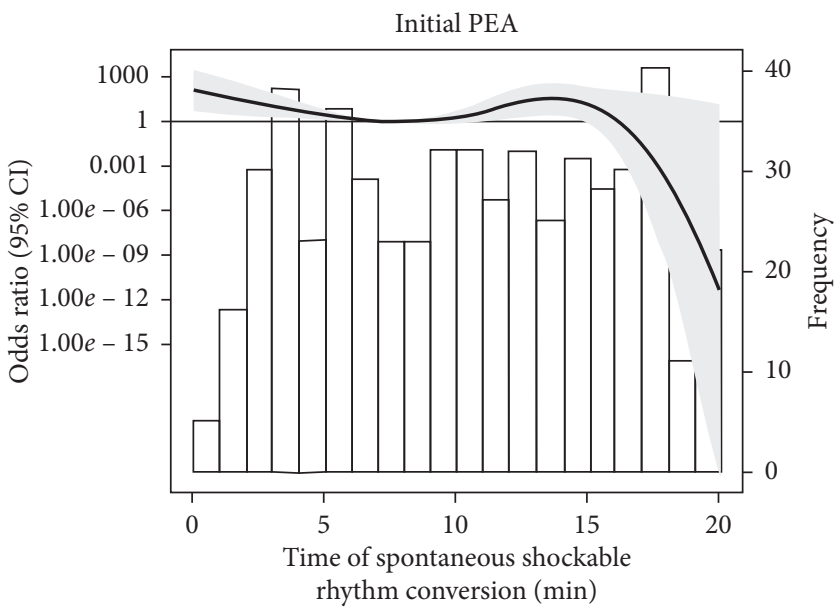

(f)

Figure 2: Adjusted odds ratios and 95\% confidence intervals comparing shockable rhythm conversion and nonshockable rhythm conversion for prehospital return of spontaneous circulation, survival to hospital discharge, and favorable functional outcome at hospital discharge by time of rhythm conversion in initial heart rhythm in the ROC Cardiac Epidemiologic Registry (version 3). (a) Adjusted odds ratios and 95\% confidence intervals for prehospital return of spontaneous circulation in OHCA patients with initial asystole or PEA. (b) Adjusted odds ratios and 95\% confidence intervals for survival to hospital discharge in OHCA patients with initial asystole or PEA. (c) Adjusted odds ratios and 95\% confidence intervals for favorable functional outcome at hospital discharge in OHCA patients with initial asystole or PEA. OHCA, out-of-hospital cardiac arrest; PEA, pulseless electrical activity; CI, confidence interval. 
beyond the first 15 min of CPR among those with initial asystole, adjusting for all covariates (Figure 2(c)). After categorizing rhythm conversion time into $<10 \mathrm{~min}$, $10-15 \mathrm{~min}$, and $\geq 15 \mathrm{~min}$ of CPR, higher odds of favorable functional outcome at discharge was observed with shockable rhythm conversion, when the initial rhythm was asystole and conversion occurred within the first $10 \mathrm{~min}$ (OR 4.28 ; $95 \%$ CI: $2.32,7.89$ ) or $10-15$ min of CPR (OR 4.38; $95 \%$ CI: 1.94, 9.90), or when the initial rhythm was PEA and conversion occurred within the first $10 \mathrm{~min}$ (OR 2.26; 95\% CI: 1.37, 3.75).

\section{Discussion}

In this retrospective analysis of 24,849 OHCA patients with initial nonshockable rhythms in a North American population-based registry, we found that shockable rhythm conversion was associated with survival and better functional outcomes at hospital discharge in patients with initial asystole, only when rhythm conversion occurred within the first 15 min of CPR. In patients with initial PEA, shockable rhythm conversion was associated with survival and better functional outcomes at hospital discharge, only when occurred within the first $10 \mathrm{~min}$ of $\mathrm{CPR}$, and the associations were weaker compared to among those with initial asystole.

The conversion from a nonshockable rhythm to a shockable rhythm in OHCA remains a subject of clinical importance. Some studies have demonstrated strong associations between shockable rhythm conversion and better outcomes in OHCA patients with initial nonshockable rhythms, whereas others did not [6-10]. Factors underlying the differing prognostic significance of shockable rhythm conversion across populations have been relatively understudied, and there has been little published data on the interactions across initial heart rhythm, rhythm conversion time, and shockable rhythm conversion in initial nonshockable rhythm OHCA. To our knowledge, only two studies have thus far analyzed data on shockable rhythm conversion and outcomes stratified by rhythm conversion time. Goto et al. studied 569,937 OHCA patients enrolled in a Japanese national registry between 2005 and 2010 [12], and Funada et al. studied 430,443 OHCA patients enrolled in the same registry between 2011 and 2014 [11]. Both studies involved only Japanese patients, categorized rhythm conversion times into $10-\mathrm{min}$ intervals, assessed outcomes at one-month post-OHCA and did not stratify analyses by initial arrest rhythm (which has previously been shown to interact with shockable rhythm conversion for its associations with OHCA outcomes) $[5,17]$. These researchers concluded that the first 20 min of CPR could be a threshold beyond which shockable rhythm conversion may no longer be associated with better outcomes in OHCA patients with initial nonshockable rhythms $[11,12]$. In contrast to these studies, the present study provides a more thorough delineation of the prognostic significance of shockable rhythm conversion stratified by initial heart rhythm, across the continuous spectrum of rhythm conversion time, and multiple OHCA outcomes that were assessed from at the field till hospital discharge.
Our findings may have clinical implications and provide a basis for the development of better CPR strategies. The current American Heart Association Guidelines for Cardiopulmonary Resuscitation and Emergency Cardiovascular Care recommend "appropriate rhythm-based strategies" for patients whose heart rhythms have evolved during CPR [18], which would indicate attempts to electrical defibrillation in patients who had undergone shockable rhythm conversions from nonshockable rhythms. However, as demonstrated in the present study, when such rhythm conversions occurred beyond certain time thresholds (i.e., $15 \mathrm{~min}$ for initial asystole and $10 \mathrm{~min}$ for initial PEA), electrical shocks may no longer confer survival or functional outcome benefits, possibly because the arresting heart had entered a "metabolic phase" where there was irreversible ischemic damage, and the heart muscles had become more susceptible to reperfusion injury [19]. Continued chest compressions to maximize circulation, in these scenarios, may therefore be preferable to electrical defibrillation attempts.

Strengths of this study include its large sample size and per-protocol ascertainment of shock delivery time and multiple OHCA outcomes. However, our study has several limitations. First, because our data originated from a North American registry, the generalizability of our findings to other populations may be limited. Second, like all observational studies, our findings may be affected by uncontrolled confounding. Nonetheless, because of the rigorous design of the ROC Cardiac Registry Epistry and its focus on per-protocol ascertainment of pertinent OHCA covariables and outcomes, we believe the influence of confounding and measurement errors was reduced to the greatest extent possible. Third, only data collected after the initiation of CPR by EMS personnel were available. We are thus unable to account for the duration of cardiac arrest or CPR performed before EMS arrival. Fourth, we did not have access to continuous heart rhythm readings and the use of first electrical shock delivery as the surrogate for shockable rhythm conversion may result in misclassifications. Further, without heart rhythm readings, we were unable to ascertain whether shockable rhythm conversions resulted in fine ventricular fibrillations, as opposed to coarse ventricular fibrillations, which are more likely to respond to electrical shocks. Nonetheless, all EMS providers participating in the ROC were instructed to adhere to clinical practice guidelines, minimizing the chances of inappropriate delivery of shocks in the absence of shockable heart rhythms.

In conclusion, the conversion from a nonshockable rhythm to a shockable rhythm was associated with better outcomes only when occurred early in initial nonshockable rhythm OHCA. These findings may facilitate the advancement of OHCA resuscitation strategies.

\section{Data Availability}

The data that support the findings of this study are openly available in the National Institutes of Health (NIH) Biological Specimen and Data Repository Information Coordinating Center (https://biolincc.nhlbi.nih.gov/studies/ roc_cardiac_epistry_3/). Requests for the date can be 
submitted through the website of the National Institutes of Health (NIH): https://biolincc.nhlbi.nih.gov/studies/ roc_cardiac_epistry_3/ (https://biolincc.nhlbi.nih.gov/ studies/rocprimed/?q=primed).

\section{Conflicts of Interest}

The authors declare that they have no conflicts of interest.

\section{Authors' Contributions}

Wanwan Zhang, Shengyuan Luo, and Daya Yang contributed equally to this work.

\section{Acknowledgments}

The authors appreciate the participants in the ROC Cardiac Epidemiologic Registry from April 2011 to June 2015 (version 3) for their contributions to this research. Dr. Xiong, as a visiting reciprocal scholar at the University of Texas Southwestern Medical Center, was supported by the Fundamental Research Funds for the Central UniversitiesTraining Funds for Young Scholars of Sun Yat-sen University (11ykpy14) from China. Dr. Idris received support from the Resuscitation Outcomes Consortium (National Heart, Lung, and Blood Institute, grant U01HL077887).

\section{References}

[1] M. K. Y. Wong, L. J. Morrison, F. Qiu et al., "Trends in shortand long-term survival among out-of-hospital cardiac arrest patients alive at hospital arrival," Circulation, vol. 130, no. 21, pp. 1883-1890, 2014.

[2] T. Kitamura, T. Iwami, T. Kawamura et al., "Nationwide improvements in survival from out-of-hospital cardiac arrest in Japan," Circulation, vol. 126, no. 24, pp. 2834-2843, 2012.

[3] P. S. Chan, B. McNally, F. Tang, and A. Kellermann, "Recent trends in survival from out-of-hospital cardiac arrest in the United States," Circulation, vol. 130, no. 21, pp. 1876-1882, 2014.

[4] M. E. Kleinman, E. E. Brennan, Z. D. Goldberger et al., "Part 5: adult basic life support and cardiopulmonary resuscitation quality," Circulation, vol. 132, no. 18 suppl 2, pp. S414-S435, 2015.

[5] S. Luo, Y. Zhang, W. Zhang, R. Zheng, J. Tao, and Y. Xiong, "Prognostic significance of spontaneous shockable rhythm conversion in adult out-of-hospital cardiac arrest patients with initial non-shockable heart rhythms: a systematic review and meta-analysis," Resuscitation, vol. 121, pp. 1-8, 2017.

[6] A. Hallstrom, T. D. Rea, V. N. Mosesso Jr et al., "The relationship between shocks and survival in out-of-hospital cardiac arrest patients initially found in PEA or asystole," Resuscitation, vol. 74, no. 3, pp. 418-426, 2007.

[7] J. Herlitz, L. Svensson, J. Engdahl, and J. Silfverstolpe, "Characteristics and outcome in out-of-hospital cardiac arrest when patients are found in a non-shockable rhythm," Resuscitation, vol. 76, no. 1, pp. 31-36, 2008.

[8] K. Kajino, T. Iwami, M. Daya et al., "Subsequent ventricular fibrillation and survival in out-of-hospital cardiac arrests presenting with PEA or asystole," Resuscitation, vol. 79, no. 1, pp. 34-40, 2008.

[9] T. M. Olasveengen, M. Samdal, P. A. Steen, L. Wik, and K. Sunde, "Progressing from initial non-shockable rhythms to a shockable rhythm is associated with improved outcome after out-of-hospital cardiac arrest," Resuscitation, vol. 80, no. 1, pp. 24-29, 2009.

[10] A. J. Thomas, C. D. Newgard, R. Fu, D. M. Zive, and M. R. Daya, "Survival in out-of-hospital cardiac arrests with initial asystole or pulseless electrical activity and subsequent shockable rhythms," Resuscitation, vol. 84, no. 9, pp. 1261-1266, 2013.

[11] A. Funada, Y. Goto, H. Tada et al., "Age-specific differences in prognostic significance of rhythm conversion from initial non-shockable to shockable rhythm and subsequent shock delivery in out-of-hospital cardiac arrest," Resuscitation, vol. 108, pp. 61-67, 2016.

[12] Y. Goto, T. Maeda, and Y. Nakatsu-Goto, "Prognostic implications of conversion from nonshockable to shockable rhythms in out-of-hospital cardiac arrest," Critical Care (London, England), vol. 18, p. 528, 2014.

[13] T. P. Aufderheide, P. J. Kudenchuk, J. R. Hedges et al., "Resuscitation outcomes consortium (ROC) PRIMED cardiac arrest trial methods. Part 1: rationale and methodology for the impedance threshold device (ITD) protocol," Resuscitation, vol. 78 , no. 2, pp. 179-185, 2008.

[14] I. G. Stiell, C. Callaway, D. Davis et al., "Resuscitation Outcomes Consortium (ROC) PRIMED cardiac arrest trial methods. Part 2: rationale and methodology for "analyze later vs. analyze early" protocol," Resuscitation, vol. 78, no. 2, pp. 186-195, 2008.

[15] T. P. Aufderheide, G. Nichol, T. D. Rea et al., "A trial of an impedance threshold device in out-of-hospital cardiac arrest," New England Journal of Medicine, vol. 365, no. 9, pp. 798-806, 2011.

[16] I. G. Stiell, G. Nichol, B. G. Leroux et al., "Early versus later rhythm analysis in patients with out-of-hospital cardiac arrest," New England Journal of Medicine, vol. 365, no. 9, pp. 787-797, 2011.

[17] R. Zheng, S. Luo, J. Liao et al., "Conversion to shockable rhythms is associated with better outcomes in out-of-hospital cardiac arrest patients with initial asystole but not in those with pulseless electrical activity," Resuscitation, vol. 107, pp. 88-93, 2016.

[18] M. S. Link, L. C. Berkow, P. J. Kudenchuk et al., "Part 7: adult advanced cardiovascular life support," Circulation, vol. 132, no. 18 suppl 2, pp. S444-S464, 2015.

[19] M. L. Weisfeldt and L. B. Becker, "Resuscitation after cardiac arrest," JAMA, vol. 288, no. 23, pp. 3035-3038, 2002. 